\title{
Life-extending dietary restriction, but not dietary supplementation of branched-chain amino acids, can increase organismal oxidation rates of individual branched-chain amino acids by grasshoppers
}

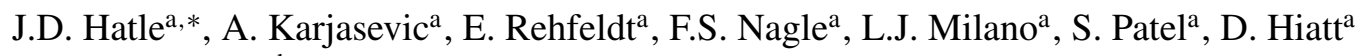 \\ and M.D. McCue ${ }^{\mathrm{b}}$ \\ ${ }^{\mathrm{a}}$ Department of Biology, University of North Florida, Jacksonville, FL, USA \\ ${ }^{\mathrm{b}}$ Sable Systems International, North Las Vegas, NV, USA
}

\begin{abstract}
.
BACKGROUND: Life-extending dietary restriction increases energy demands. Branched-chain amino acids (BCAAs), at high levels, may be detrimental to healthspan by activating the mechanistic Target of Rapamycin (mTOR). Whether organismal oxidation of BCAAs increases upon dietary restriction is unknown.

OBJECTIVE: Test whether dietary restriction (DR, which creates an energy deficit) or supplemental dietary BCAAs (superfluous BCAAs) increases oxidation of BCAAs, potentially reducing their levels to improve healthspan.

METHODS: Grasshoppers were reared to middle-age on one of four diets, each a level of lettuce feeding and a force-fed solution: 1) ad libitum lettuce \& buffer, 2) ad libitum lettuce \& supplemental BCAAs, 3) DR lettuce \& buffer, and 4) DR lettuce \& supplemental BCAAs. On trial days, grasshoppers were force-fed one ${ }^{13} \mathrm{C}-1$-BCAA (isoleucine, leucine, or valine). Breath was collected and tested for ${ }^{13} \mathrm{CO}_{2}$, which represents organismal oxidation of the amino acid. Additional trials re-tested oxidation of leucine (the most potent activator of mTOR) in both females and males on dietary restriction.

RESULTS: Dietary restriction generally increased cumulative oxidation of each BCAA in females and hungry males over $\sim 8 \mathrm{hr}$. Results were consistent for isoleucine and valine, but less so for leucine. Supplementation of BCAAs, in combination with dietary restriction, increased isoleucine in hemolymph, with similar trends for leucine and valine. Despite this, supplementation of BCAAs did not alter oxidation of any BCAAs.

CONCLUSIONS: Dietary restriction can increase oxidation of BCAAs, likely due to an energy deficit. The increased oxidation may decrease available BCAAs for activation of mTOR and improve healthspan.
\end{abstract}

Keywords: Aging, metabolism, hunger, isoleucine, leucine, valine

\section{Introduction}

*Corresponding author: J.D. Hatle, Department of Biology, University of North Florida, 1 UNF Drive, Jacksonville FL, USA 32224. Tel.: +1 904620 2778, Fax: +1 904620 3885; E-mail: jhatle@unf.edu.
Dietary restriction extends lifespan in many species, particularly in females. Dietary protein needs to be restricted to extend lifespan, and this consensus has developed over the past decade from experiments 
using the Geometric Framework approach [e.g., 13 , but see 4]. This has led to a heightened interest in the biochemical and physiological roles of specific groups amino acids, both their dietary levels and metabolism [5, 6].

Numerous studies have revealed important relationships among amino acid feeding, amino acid metabolism, and longevity. First, restriction of only methionine can extend lifespan [e.g., 7, 8], by altering the transulfuration pathway and stimulating cellular $\mathrm{H}_{2} \mathrm{~S}$ production $[9,10]$. Second, tryptophan restriction may extend lifespan in rodents [6]. Third, diets with a composition of amino acids that matches the amino acids in the exome can extend lifespan in flies, while eating less but developing faster [11]. Last, dietary restriction of the branched-chain amino acids (BCAAs) isoleucine, leucine, and valine extends lifespan in flies, but so does restriction of three other essential amino acids [12]. The catabolism of BCAAs can regulate aging in worms [13]. Dietary restriction of BCAAs may also act through anti-diabetic effects, as it can improve weight control and insulin sensitivity in mice [14]. Here, we focus on organismal oxidation of the BCAAs upon life-extending dietary restriction or dietary supplementation of BCAAs.

Classic protocols for life-extending dietary restriction limit either the food quantity (e.g., for mice) or the food quality (e.g., for flies). Both result in a hungry animal, as shown by increased expression of neuropeptide Y/F $[15,16]$. Dietary restriction is distinct from starvation, but nonetheless the classic dietary restriction protocols typically create an energy deficit. This deficit is concurrent with active AMPK (5' adenosine monophosphate-activated protein kinase), which can help extended lifespan [17]. An energy deficit could increase the need to oxidize any ingested macronutrient, including BCAAs, for production of ATP (adenosine triphosphate).

Reduced BCAA levels, due to increased oxidation for ATP, could improve healthspan in at least two ways. First, BCAAs are the most potent amino acids at stimulating the mechanistic target of rapamycin (mTOR) pathway, with leucine being the most powerful [e.g., 18]. Inhibition of the mTOR pathway, either via genetic or pharmacological approaches, increases lifespan in a wide range of animal species [e.g., 19]. Reducing the BCAAs that are available to activate mTOR may also reduce the mTOR signal and improve healthspan. Increased oxidation of BCAAs for ATP production should, in theory, reduce cellular levels of BCAAs. Sec- ond, increased BCAAs levels are associated with the onset of diabetes [e.g., 20]. Switching mice to a diet very low in BCAAs reduced weight and improved insulin sensitivity [21]. While reduced BCAAs levels in animals might improve healthspan by other mechanisms, these two are the most likely candidates.

Romalea microptera (the Eastern lubber grasshopper; hereafter 'grasshopper') females are a model for studying the role of organismal oxidation of nutrients in the context of aging [22]. First, reducing diet quantity by $30 \%$ cuts reproduction in half and extends lifespan $\sim 15 \%$ [e.g., 23, 24]. Second, adult consumption of dietary protein is required for egg production. Female grasshoppers, despite thriving on a plant diet, produce large clutches of eggs that weigh $\sim 10-20 \%$ of the entire insect [e.g., 24], and one-half the mass of the clutch is vitellin (egg yolk protein) [e.g., 25]. Third, the large size of grasshoppers $(\sim 8 \mathrm{~g}$ gravid) relative to other insects facilitates experimental manipulations and metabolic measures. For example, individuals can be force-fed $40 \mu \mathrm{l}$ in a feeding, and the ${ }^{13} \mathrm{CO}_{2}$ in breath can be measured from a single individual [26]. Last, these grasshoppers have a relatively long lifespan [e.g., 24]. They are welldefended against bird predators [27], and species with low predation rates (e.g., clams) typically have long lifespans [e.g., 28]. Using dietary restriction to extend lifespan of an already long-lived species is notable.

Prior work showed that oxidation of leucine can be increased upon dietary restriction in female lubber grasshoppers [26]. In that study we tracked a representative of each macronutrient (leucine for protein, glucose for carbohydrate, and oleic acid for lipids) from ingestion to oxidation or allocation to various tissues. Surprisingly, we found that dietary restriction increased leucine oxidation but not glucose or oleic acid oxidation, even during egg production.

Here, we test whether diet quantity restriction or dietary supplementation of BCAAs increases the organismal oxidation of each of the BCAAs. Our broad hypothesis is that the energy deficit of dietary restriction increases oxidation of BCAAs, which reduces the amount of BCAAs available for other functions and thereby contributes to increased healthspan. Specifically, we test the predictions that: 1) diet quantity restriction increases the rate of oxidation of each BCAA, due to energy demands; 2) dietary supplementation of BCAAs increases their levels in the hemolymph, increases feeding rate, and increases egg production; and 3) dietary supplemen- 
tation of BCAAs increases the rate of oxidation of each BCAA, due to superfluous BCAAs. We find that dietary restriction, but not supplemental BCAAs, typically increases oxidation of BCAAs in females and young males, and that supplementation of BCAAs does not alter feeding or egg production.

\section{Materials and Methods}

\subsection{Effects of dietary restriction and supplementation of BCAAs on oxidation of $B C A A s$ in females (Experiment 1)}

Juvenile male and female lubber grasshoppers were raised in communal metal cages $\left(0.027 \mathrm{~m}^{3}\right)$ and were fed Romaine lettuce daily, and wheat germ, green beans, and carrot tops occasionally. The day of the molt to adult, females were isolated, placed into individual $500 \mathrm{~cm}^{2}$ cages, and were reared at $35^{\circ} \mathrm{C}$ during a 14-hr photophase and $27^{\circ} \mathrm{C}$ during a $10-\mathrm{hr}$ scotophase.

\subsubsection{Lettuce diets}

For the first 17 days of adulthood, all females were fed Romaine lettuce ad libitum. At 18 days after adult molt, individuals were serially assigned to one of two lettuce feeding groups: A) ad libitum lettuce, or B) dietary restriction (DR; $60 \%$ of the average lettuce consumed by ad libitum grasshoppers). This DR protocol is known to extend lifespan [e.g., 23]. Each week the lettuce ingested by the ad libitum group was measured, and the amount offered to the DR group was adjusted as needed, as in several previous studies [e.g., 29].

\subsubsection{Forced feeding of branched-chain amino acids}

At 25 days after the final adult molt, grasshoppers were started on daily force-feeding treatments (either a phosphate buffered saline [a control] or a mixture of the three BCAAs). These liquid diet feedings resulted in four treatment groups (initial $n=32$ per group): ad libitum lettuce \& BCAAs, ad libitum lettuce \& buffer, DR lettuce \& BCAAs, and DR lettuce $\&$ buffer (Supplemental Figure 1).

The amount of BCAAs to be force-fed was set so that the total BCAAs consumed by the DR \& BCAA group was equal to the total BCAAs consumed by the ad libitum \& buffer group. One gram of Romaine lettuce contains $0.45 \mathrm{mg}$ isoleucine. $0.76 \mathrm{mg}$ leucine, and $0.55 \mathrm{mg}$ valine [30]. To prepare our standard solution of BCAAs, $45 \mathrm{mg}$ isoleucine, $76 \mathrm{mg}$ leucine, and $55 \mathrm{mg}$ valine were placed in a $20-\mathrm{ml}$ glass vial, and $3.5 \mathrm{ml}$ of $\mathrm{pH} 5$ phosphate buffered saline was added. The BCAAs are among the least water-soluble amino acids, so dissolving them in the phosphate buffered saline required heating to $\sim 70^{\circ} \mathrm{C}$ and mixing. This created a solution that contained the same ratio of the three BCAAs as contained in Romaine lettuce. Initially, the solutions were prepared weekly, frozen for storage, and thawed and resuspended before use. Starting at about 40 days, solutions were prepared daily.

The volume of the solution of BCAAs that was force-fed to each individual (in the ad libitum \& BCAA and DR \& BCAA groups) was adjusted weekly when DR meal sizes were calculated. Again, the amount of BCAA force-fed was set so that the total amount of BCAAs consumed by the DR \& BCAA group was equal to the total amount of BCAAs consumed by the ad libitum \& buffer group. A typical difference between ad libitum lettuce feeding and DR lettuce feeding was 2.0 wet grams. This required force-feeding $70 \mu \mathrm{l}$ of our standard solution of BCAAs daily, containing $0.90 \mathrm{mg}$ isoleucine, $1.52 \mathrm{mg}$ leucine, and $1.10 \mathrm{mg}$ valine. We force-fed liquids to grasshoppers using a $10 \mu$ l pipette [e.g., 26]. Each individual usually can consume three or four $10 \mu \mathrm{l}$ aliquots per meal. Force-feedings were conducted every morning and late afternoon to deliver the required daily volume.

\subsubsection{Concentrations of BCAAs in hemolymph}

Hemolymph samples were collected via a neck prick from six selected individuals per group, when the grasshoppers were about 75 days into adulthood. Samples were collected for two consecutive days to obtain a total of $60 \mu \mathrm{l}$ from each individual. Sampling was prior to the daily morning feeding. Hemolymph samples were sent to the University of Florida Metabolomics lab and were analyzed for amino acids via liquid chromatography and mass spectroscopy.

\subsubsection{Oviposition testing}

Oviposition was tested three times a week [e.g,. 24]. Each grasshopper was placed into an individual 1-liter cup filled about $3 / 4$ with slightly damp sand and covered with a vented lid. On testing days, every individual received the force feeding and then was placed in its sand cup for about $1 \mathrm{hr}$ to permit probing. Indi- 
viduals that showed signs of probing were left in their sand cup until the next morning and skipped that day's feedings. The following day eggs were counted, and the number of eggs and date of laying was recorded. The combination of age at oviposition and number of eggs laid captures most of the dietary effects in reproductive investment [e.g., 24].

\subsubsection{Organismal oxidation of BCAAs}

At about 80 days into adulthood, grasshoppers were tested for oxidation of each BCAA. Each individual was tested for oxidation of only one BCAA, to avoid the possibility of accumulation of the ${ }^{13} \mathrm{C}$ tracer. On test days, prior to the lettuce feeding, an individual was force-fed the prescribed daily dosage of all three BCAAs. One of the three BCAAs was labeled with ${ }^{13} \mathrm{C}$; the other two amino acids were not enriched in ${ }^{13} \mathrm{C}$, thereby allowing tracking of the ${ }^{13} \mathrm{C}$-labeled BCAAs. Each tracer was the $1-{ }^{13} \mathrm{C}$ $\mathrm{L}$ isoform (e.g., $1{ }^{1}{ }^{13} \mathrm{C}$-L-leucine). The dosages of the ${ }^{13} \mathrm{C}$-labeled BCAAs were: $342 \mu \mathrm{g}$ isoleucine, or $583 \mu \mathrm{g}$ leucine, or $416 \mu \mathrm{g}$ valine. These tests took place over about 2 weeks, and the amounts of force-fed BCAAs were held constant during this period.

Immediately after force-feeding the tracer, an individual was placed in a $100 \mathrm{ml}$ gas-tight syringe for about 60 minutes to collect the exhaled breath, after which about $20 \mathrm{~mL}$ of the gas was collected and transferred to a $12-\mathrm{mL}$ evacuated vial [e.g., 26]. The $100 \mathrm{ml}$ syringe was then ventilated with fresh air and the grasshopper was returned to the syringe, which was again sealed. Breath (gas) samples were collected at 1, 2, 3, 4, 6, 8, and 13 hours after they received the ${ }^{13} \mathrm{C}$ tracer amino acid. The measurement of ${ }^{13} \mathrm{C}$ in the exhaled $\mathrm{CO}_{2}$ and the calculation of the rate of tracer oxidation was done as previously described [31, 32], using non-dispersive infrared spectroscopy.

\subsubsection{Quantification of hexamerin storage proteins}

Hexamerins are a class of storage proteins that are found in arthropods, and the levels of hexamerins in the hemolymph are a good measure of protein storage within the individual [33]. Hemolymph samples (collected during dissections) were separated by native polyacrylamide gel electrophoresis, which separates the three hexamerins in the hemolymph of lubber grasshoppers [34]. Each gel included a standard curve using three concentrations of bovine serum albumin, in addition to eight hemolymph samples, and was run for $\sim 13$ hr at $4{ }^{\circ} \mathrm{C}$ and $150 \mathrm{~V}$. The gels were then stained with Coomassie Brilliant Blue R250. The density of each band was analyzed using ImageJ. The top point on the standard curve was set as the maximum possible amount of hexamerins, to avoid skewed data.

\subsection{Effects of dietary restriction on organismal oxidation of leucine in females (Experiment 2 )}

We conducted further tests on the oxidation of leucine, the most powerful BCAA for activation of the mTOR pathway. Grasshoppers were obtained and reared to adulthood as in the previous year, except the rearing temperature was reduced to $32^{\circ} \mathrm{C}$ during the day and $24^{\circ} \mathrm{C}$ during the night, to better separate developmental events.

Adult females were isolated and reared on ad libitum lettuce for 18 days (total $n=28$ ). They were serially assigned to two feeding treatments: ad libitum Romaine lettuce or Dietary Restriction lettuce (60\% of that consumed by the ad libitum group). After 39 days on these diets, adult females were tested for leucine oxidation as per the previous experiment, using a dosage of $619 \mu \mathrm{g}$ of $1-{ }^{13} \mathrm{C}$-L-leucine as the tracer. Completing all these trials required 3 days (age of testing $=57 \pm 2$ days). Then all individuals were reciprocally switched to the other diet treatment: that is, those fed ad libitum from days 19 to 57 were switched to dietary restriction, and those fed on dietary restriction from days 19 to 57 were switched to ad libitum lettuce. Grasshoppers were kept on these diets for 41 days and then tested again for leucine oxidation (at $95 \pm 2$ days).

\subsection{Organismal oxidation of leucine in males (Experiment 3)}

Male lubber grasshoppers have never been tested for possible effects of dietary restriction on lifeextension. Despite this, males can be used to test whether dietary restriction that creates an energy deficit increases oxidation of leucine. A pilot study using young adult males identified the lowest level of feeding that avoided mortality due to starvation. Young males on 0.1 or $0.2 \mathrm{~g}$ of lettuce per day showed high mortality, while males on 0.3 to $0.5 \mathrm{~g}$ of lettuce per day had lower (minimal) mortality ( $t$-test, $P=0.012$ ). Therefore, we chose 0.4 or $0.5 \mathrm{~g}$ per day as the dietary restriction quantity (i.e., the lowest feeding rate that avoids starvation). We later estimated this to be $30 \%$ of ad libitum feeding. 
Males were reared from adult molt to the testing age on either dietary restriction or ad libitum lettuce. At median age 14 days into adulthood (range $7-19$ days), males were tested for leucine oxidation, using $150 \mu \mathrm{g}$ of $1{ }^{13} \mathrm{C}$-L-leucine as the tracer.

In an additional trial with males, starting at $76 \pm 1$ days into adulthood, grasshoppers were reared on one of the three diet treatments: A) $0.4 \mathrm{~g}$ per day, B) $0.88 \mathrm{~g}$ per day, or C) ad libitum lettuce. Feeding rates may have changed from young to middle-age, so we do not have an estimate of the percentages of ad libitum feeding for these trials. After $21 \mathrm{~d}$ on the respective diet treatments, the middle-aged males also were tested for leucine oxidation using $150 \mu \mathrm{g}$ of $1-{ }^{13} \mathrm{C}$-L-leucine.

\subsection{Statistics}

Data with two treatment levels (e.g., lettuce level and BCAA supplementation) and multiple measures from the same individual (e.g., maximum rate of oxidation, maximum time of oxidation, and Area Under the Curve) were analyzed by two-way MANOVA, with REGWQ post-tests. The feeding data (Ad lib \& BCAA vs Ad lib \& buffer) was tested with a repeated measures ANOVA. Oxidation curves were analyzed with MANOVA with time as a dependent variable. All these analyses were run in SAS. The association of the time of maximum leucine oxidation and the timing of clutch laying was tested by fitting a straight line to the data and determining the $\mathrm{R}^{2}$ value, using Excel. The oxidation parameters for young males, with only one experimental level (i.e., feeding rate), were tested with Student's t-tests, with Bonferroni's correction of alpha.

\section{Results}

\subsection{Experiment 1}

\subsubsection{Hemolymph levels of BCAAs were slightly increased by force-feeding upon DR lettuce}

Hemolymph levels of valine were $\sim 3$-fold higher than leucine or isoleucine, which is consistent with previous data for other grasshoppers [25], and supports the accuracy of the analysis. The general trend was for force-feeding of BCAAs to increase hemolymph levels of BCAAs, but only when on DR lettuce (Fig. 1). Hemolymph levels of BCAAs (total $n=24$ ) were slightly affected by force-feeding (two-way MANOVA; Pillai's Trace $\mathrm{F}_{3,18}=2.95$;

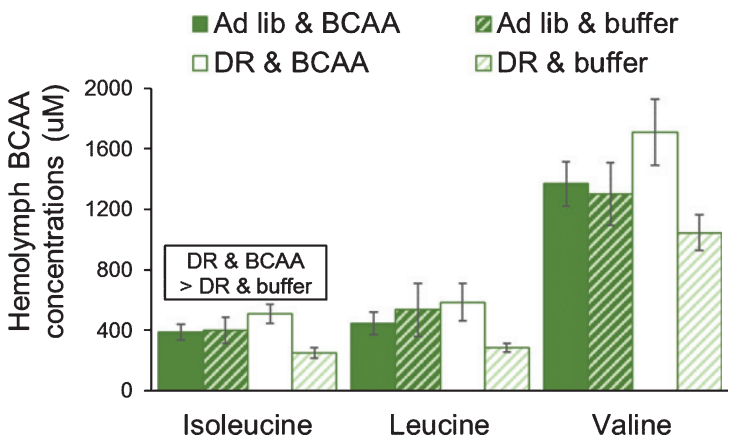

Fig. 1. Force-feeding of BCAAs tended to increase BCAAs in hemolymph of female grasshoppers fed on dietary restriction lettuce (60\% of ad libitum). Grasshoppers were force-fed BCAAs daily: approximately $0.90 \mathrm{mg}$ isoleucine, $1.52 \mathrm{mg}$ leucine, and $1.10 \mathrm{mg}$ valine. The amount of BCAAs fed was adjusted weekly so that the total consumption of BCAAs was the same in the ad libitum lettuce $\&$ buffer and DR lettuce \& BCAA groups. Data were tested using a two-way MANOVA. Error bars show one Standard Error.

$P=0.060)$, but were not affected by the level of lettuce feeding (Pillai's Trace $\mathrm{F}_{3,18}=0.30 ; P=0.827$ ).

Isoleucine levels in the hemolymph were 2 -fold higher in the DR \& BCAA group than in the DR \& buffer group $(P=0.036)$, as supported by a significant interaction of lettuce diet and force-feeding for isoleucine $\left(\mathrm{F}_{3,23}=4.72 ; P=0.042\right)$. Leucine levels in the hemolymph were not significantly affected by lettuce diet or force-feeding of BCAAs. However, the non-significant trends were similar in direction to changes in isoleucine. Hemolymph levels of leucine were 2-fold higher in the DR \& BCAA group than in the DR \& buffer group $(P=0.273)$, and the interaction of lettuce diet and force-feeding approached statistical significance $\left(F_{2,23}=2.96\right.$; $P=0.101)$. Likewise, valine levels in the hemolymph were not significantly affected by lettuce diet or force-feeding of BCAAs, yet valine levels were $63 \%$ higher in the DR \& BCAA group than in the DR \& buffer group $(P=0.065)$, and the interaction of lettuce diet and force-feeding approached statistical significance $\left(F_{2,23}=2.89 ; P=0.105\right)$. Overall, these results suggest that our chronic force-feeding of BCAAs was effective, and it tended to increase level of BCAAs in grasshoppers on DR lettuce.

\subsubsection{Force-feeding of BCAAs did not alter feeding rate or clutch size}

The amount of lettuce eaten by both of the ad libitum-fed lettuce groups was measured weekly. 


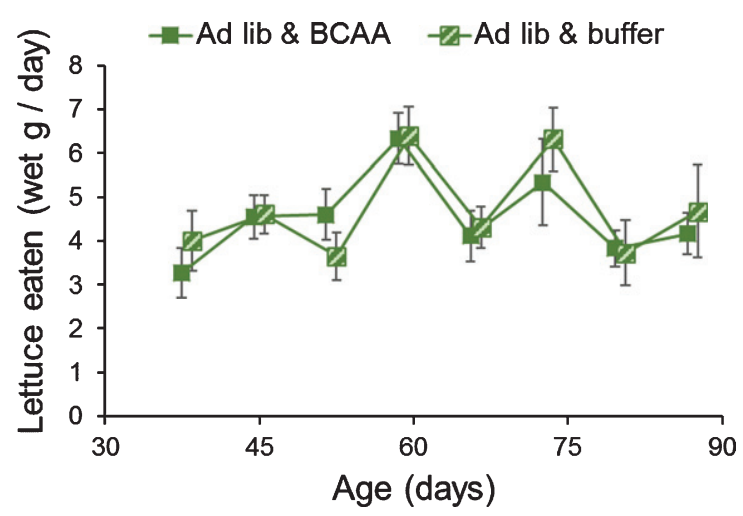

Fig. 2. Dietary supplementation of BCAAs did not alter lettuce feeding in grasshoppers. Adult female grasshoppers were reared on ad libitum lettuce \& BCAA or on ad libitum lettuce \& buffer. Age refers to days after adult molt. Feeding rates varied week to week, but not due to supplementation of BCAAs. Data were tested via one-way repeated-measures ANOVA. Data are offset to show \pm 1 Standard Error bars.

Dietary supplementation of BCAAs did not alter feeding rate in any week (one-way repeated-measures ANOVA; all $P>0.05$; Fig. 2). In contrast to the lack of effect of BCAAs on feeding, there was a significant effect of time on lettuce feeding (Pillai's Trace $\mathrm{F}_{7,16}=5.68, P=0.002$ ), suggesting feeding rates differed on the different days tested.

Clutch 1 contained significantly more eggs than clutch 2 (two-way MANOVA; Pillai's Trace $\mathrm{F}_{1,97}=7.82 ; P=0.0062$; Fig. 3 ), as in previous work (e.g., Drewry et al. 2011). The number of eggs laid upon DR lettuce was reduced $28 \%$ and $39 \%$ in clutch 1 and clutch 2 , respectively (both $P<0.001$ ). In contrast, the number of eggs laid upon supplementation of BCAAs in clutch 1 and clutch 2 was not changed (both $P>0.4$ ).

\subsubsection{Dietary restriction increased organismal oxidation of BCAAs, but supplemental $B C A A s$ did not}

Isoleucine oxidation was significantly affected by the interaction of time after tracer ingestion and lettuce feeding (two-way MANOVA with time as a dependent variable; Pillai's Trace $\mathrm{F}_{6,27}=2.81$; $P=0.030$; Fig. 4A). Specifically, grasshoppers on DR lettuce had greater rates of oxidation of isoleucine at 2, 3, and $4 \mathrm{hrs}$ after tracer ingestion (all $P<0.05$ ); these are the times after tracer ingestion with the greatest rates of oxidation. In contrast to lettuce feeding, supplementation of BCAAs had no effect on rates of isoleucine oxidation during hours 1 through 8. Supplementation of BCAAs decreased isoleucine

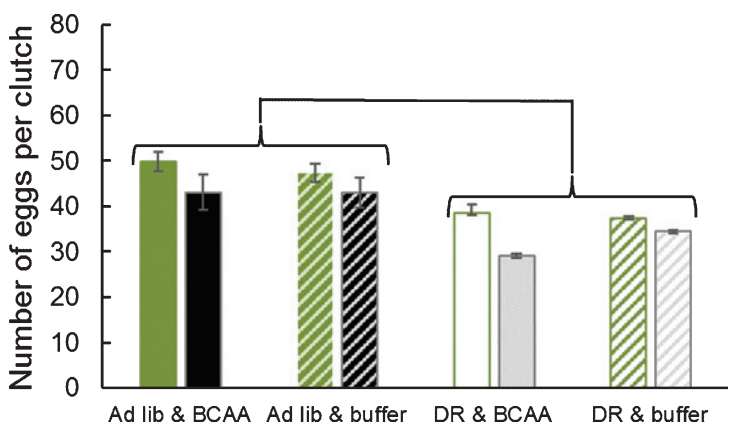

Fig. 3. Number of eggs per clutch was reduced by life-extending dietary restriction, but was not affected by supplemental dietary BCAAs. Green-shaded bars represent the first clutch, while blackor gray-shaded bars represent the second clutch. Adult female grasshoppers were reared on either ad libitum or dietary restriction lettuce and supplemented with BCAAs or buffer. Data were tested with a two-way MANOVA. Error bars show one Standard Error.

oxidation at $13 \mathrm{hr}(P=0.038)$, when oxidation rates were low in all treatment groups.

Leucine oxidation was not significantly affected by the interaction of time after tracer ingestion and lettuce feeding (two-way MANOVA with time as a dependent variable; Pillai's Trace $\mathrm{F}_{6,24}=1.67$; $P=0.171$; Fig. 4C). That is, leucine oxidation was not increased by DR lettuce feeding at any time (all $P>0.24$ ). Leucine oxidation was $\sim 50 \%$ higher upon DR lettuce feeding (but not significant). Supplementation of BCAAs also had no effect on leucine oxidation (all $P>0.065$ ), although oxidation was nearly decreased at $4 \mathrm{hr}$ and nearly increased at $8 \mathrm{hr}$.

Valine oxidation was significantly affected by interaction of time after tracer ingestion and lettuce feeding (two-way MANOVA with time as a dependent variable; Pillai's Trace $\mathrm{F}_{6,24}=2.48 ; P=0.047$; Fig. 4E). Specifically, grasshoppers on DR lettuce had greater oxidation of valine at 2, 3, 4, and $6 \mathrm{hrs}$ after tracer ingestion (all $P<0.05$ ). In contrast to lettuce feeding, supplementation of BCAAs had no effect on valine oxidation at any period (all $P>0.34$ ).

With these same data, we analyzed aspects of the oxidation curves, specifically the magnitude of the maximum rates of oxidation, the time at which maximum oxidation occurs, and the Area Under the Curve (AUC; which represents the cumulative tracer oxidation). For isoleucine, lettuce feeding (two-way MANOVA, Pillai's Trace $F_{3,30}=3.20$; $P=0.037$; Fig. 4B) but not BCAA supplementation $\left(\mathrm{F}_{3,30}=1.01 ; P=0.400\right)$, affected oxidation parameters. Specifically, the maximum rate $\left(F_{3,35}=3.31\right.$; 

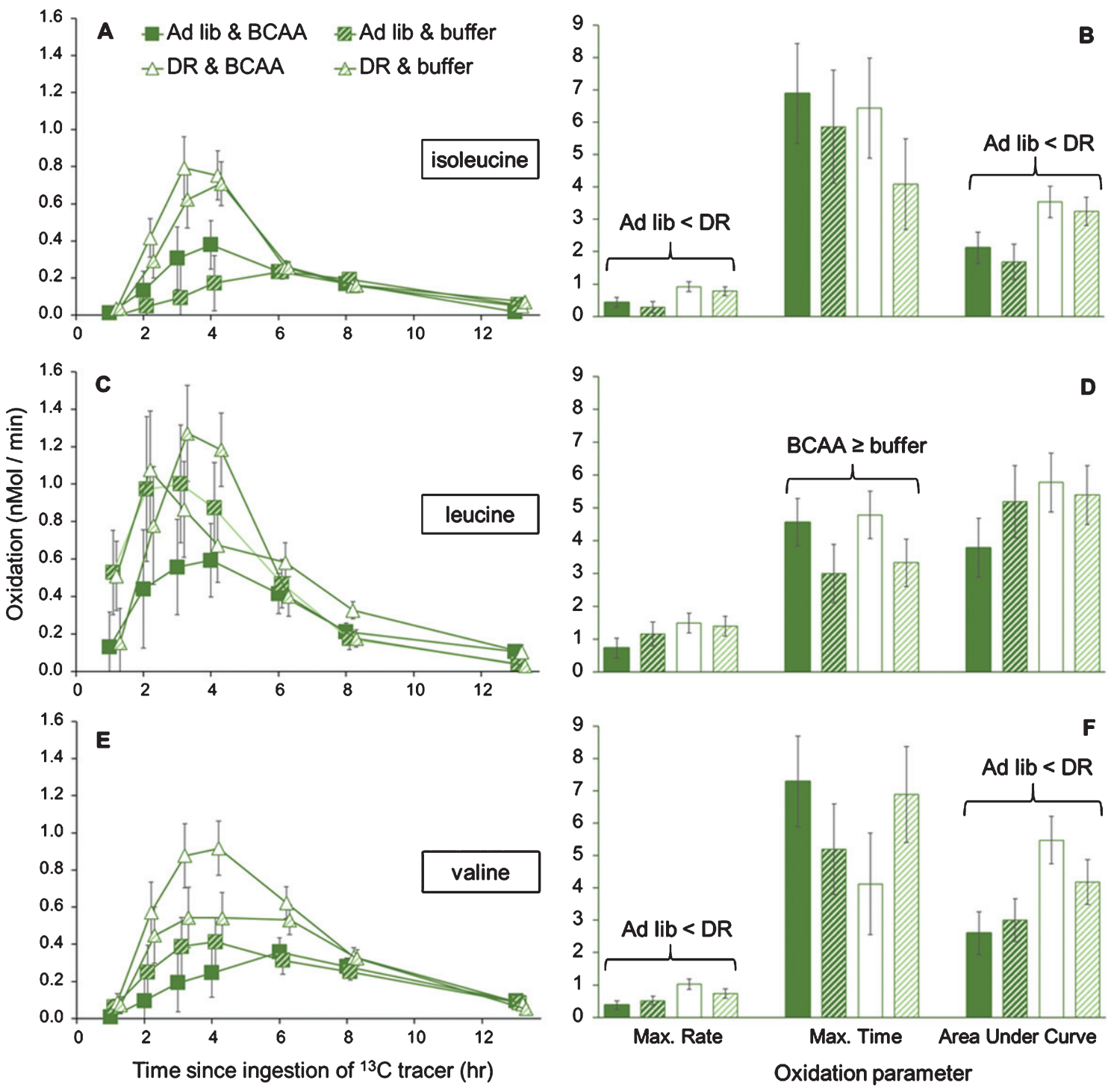

Fig. 4. Organismal oxidation of individual BCAAs upon ad libitum or DR lettuce feeding and supplementation with BCAAs or buffer, in female grasshoppers. Panels A and B show oxidation of isoleucine, panels C and D show oxidation of leucine, and panels E and F show oxidation of valine. Life-extending dietary restriction of lettuce increased oxidation of isoleucine and valine at the periods of greatest oxidation, with significant changes in the maximum rate of oxidation and the cumulative oxidation (i.e., Area Under the Curve), as shown by two-way MANOVA. Dietary restriction did not alter oxidation of leucine, though BCAA supplementation tended to result in earlier oxidation of leucine. For panels $\mathrm{B}, \mathrm{D}$, and F, maximum rates are $\mathrm{nMol} / \mathrm{min}$, maximum times are hours after tracer ingestion, and Area Under the Curve is in $\mathrm{nMol} / \mathrm{min} * 100$. Trials were run at middle-age, $\sim 90$ days into adulthood. Data are offset to show standard error bars.

$P=0.032)$ and the cumulative oxidation $\left(\mathrm{F}_{3,35}=3.18\right.$ $P=0.037)$ were increased upon DR lettuce, while time of maximum oxidation was not $\left(\mathrm{F}_{3,35}=0.72\right.$; $P=0.549$ ).

For leucine, neither lettuce feeding (two-way MANOVA, Pillai's Trace $\mathrm{F}_{3,27}=1.61 ; P=0.201$; Fig. 4D) nor BCAA supplementation $\left(\mathrm{F}_{3,27}=1.68\right.$; $P=0.194)$, affected oxidation parameters. Supple- mentation of BCAA marginally increased the time of maximum oxidation of leucine $(P=0.060)$, but the main effect was non-significant $\left(\mathrm{F}_{3,32}=1.30\right.$; $P=0.294)$, reducing confidence in the result.

For valine, lettuce feeding (two-way MANOVA, Pillai's Trace $\mathrm{F}_{3,31}=3.20 ; P=0.042$; Fig. $4 \mathrm{~F}$ ) but not BCAA supplementation $\left(\mathrm{F}_{3,31}=0.16 ; P=0.926\right)$, affected oxidation parameters. As for isoleucine, the 


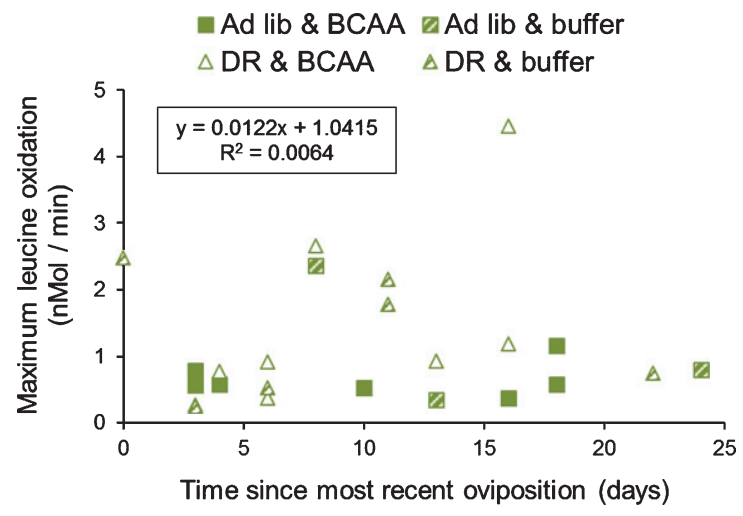

Fig. 5. Association of organismal oxidation of leucine and time since most recent oviposition, in grasshoppers. Grasshoppers were reared upon ad libitum or DR lettuce feeding and supplementation with BCAAs or buffer. There was no association of the amount of BCAA oxidized and the moment in the grasshopper's egg production cycle $\left(\mathrm{R}^{2}=0.0064\right.$, using Excel curve fitting).

cumulative oxidation $\left(\mathrm{F}_{3,36}=3.38 ; P=0.030\right)$ and the maximum rate of oxidation was increased upon DR lettuce $\left(\mathrm{F}_{3,36}=3.54 ; P=0.025\right)$, while the maximum time of oxidation was not $\left(\mathrm{F}_{3,36}=0.99 ; P=0.401\right)$.

\subsubsection{Inter-individual variation in maximum rates of leucine oxidation is not due to clutch cycling}

Oxidation of leucine showed great variation across individuals. We examined whether this variation was due to the point in the clutch cycle at which the individual was tested. These grasshoppers lay serial clutches, with the first clutch laid at $\sim 40$ days after adult molt, and subsequent clutches laid every $\sim 20$ days [e.g., 24]. In addition, previous work has shown that hemolymph levels of reproductive proteins and hormones, and the mass of the fat body, all peak 10-14 days before laying the first clutch. Therefore, we examined the relationship between the maximum level of leucine oxidation and the time since the most recent oviposition (total $n=24$ ). There was no association between the moment within the clutch cycle and the maximum level of leucine oxidation by an individual grasshopper $\left(\mathrm{R}^{2}=0.0064\right.$; Excel curve fitting; Fig. 5).

\subsubsection{Levels of hemolymph storage proteins were not altered by supplementation of BCAAs}

Upon dissection at age $\sim 100$ days into adulthood, hemolymph samples (total $n=24$ ) were collected and later tested for levels of hexameric storage proteins [as per 34]. Levels of all storage proteins

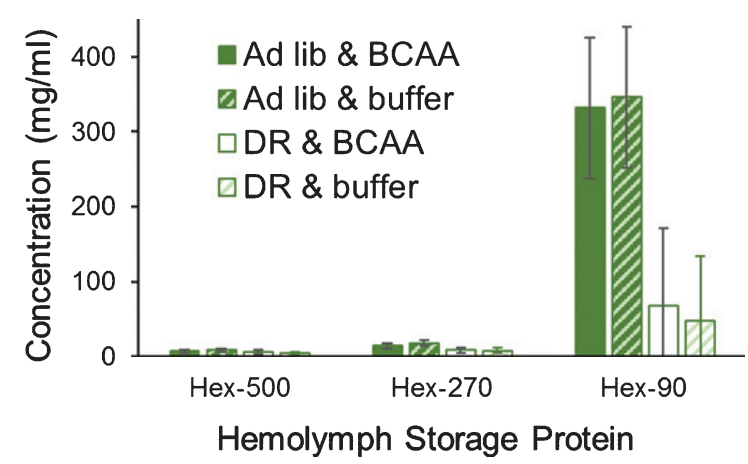

Fig. 6. Levels of three hexameric storage proteins from the hemolymph of grasshoppers. Grasshoppers were reared upon ad libitum or DR lettuce feeding and supplementation with BCAAs or buffer. Levels of hexamerin-90 and hexamerin-270, but not hexamerin-500, were significantly reduced by a dietary restriction known to extend lifespan. Data were tested via two-way MANOVA. Error bars are one Standard Error.

were significantly reduced by DR lettuce feeding (two-way MANOVA; Pillai's Trace $\mathrm{F}_{3,18}=6.68$; $P=0.003$; Fig. 6). Most important, storage protein levels were not affected by supplementation of BCAAs $\left(\mathrm{F}_{3,18}=0.13 ; P=0.941\right)$. Hexamerin-90 (an arylphorin rich in aromatic amino acids) makes up about $70 \%$ of the three storage proteins in the hemolymph of lubber grasshoppers [34]. Levels of hexamerin-90 were significantly reduced by DR lettuce $(P=0.007)$. Levels of hexamerin-270 were barely reduced by DR lettuce $(P=0.050)$, and levels of hexamerin-500 were not affected by lettuce feeding $(P=0.242)$. These responses of hexamerins to DR lettuce feeding are consistent with previous work (Hathaway et al. 2009).

\subsection{Dietary restriction can, but does not always, increase leucine oxidation}

In Experiment 2, leucine oxidation was re-tested in middle-aged, adult females (total $n=28$ ). This experiment used a counterbalanced design, in which each individual was tested on both ad libitum lettuce and DR lettuce, separated by $\sim 40 \mathrm{~d}$. The trials at $\sim 57 \mathrm{~d}$ and $\sim 95 \mathrm{~d}$ gave quite different results, so we analyzed them separately.

In the trials at $\sim 57 \mathrm{~d}$, leucine oxidation was not affected by the interaction of time after tracer ingestion and lettuce feeding (one-way MANOVA with time as a dependent variable; Pillai's Trace $\mathrm{F}_{7,20}=1.24 ; P=0.327$; Fig. $\left.7 \mathrm{~A}\right)$. That is, in this trial, DR lettuce did not alter leucine oxidation at any time tested. In contrast, in the trials as $\sim 95 \mathrm{~d}$, 

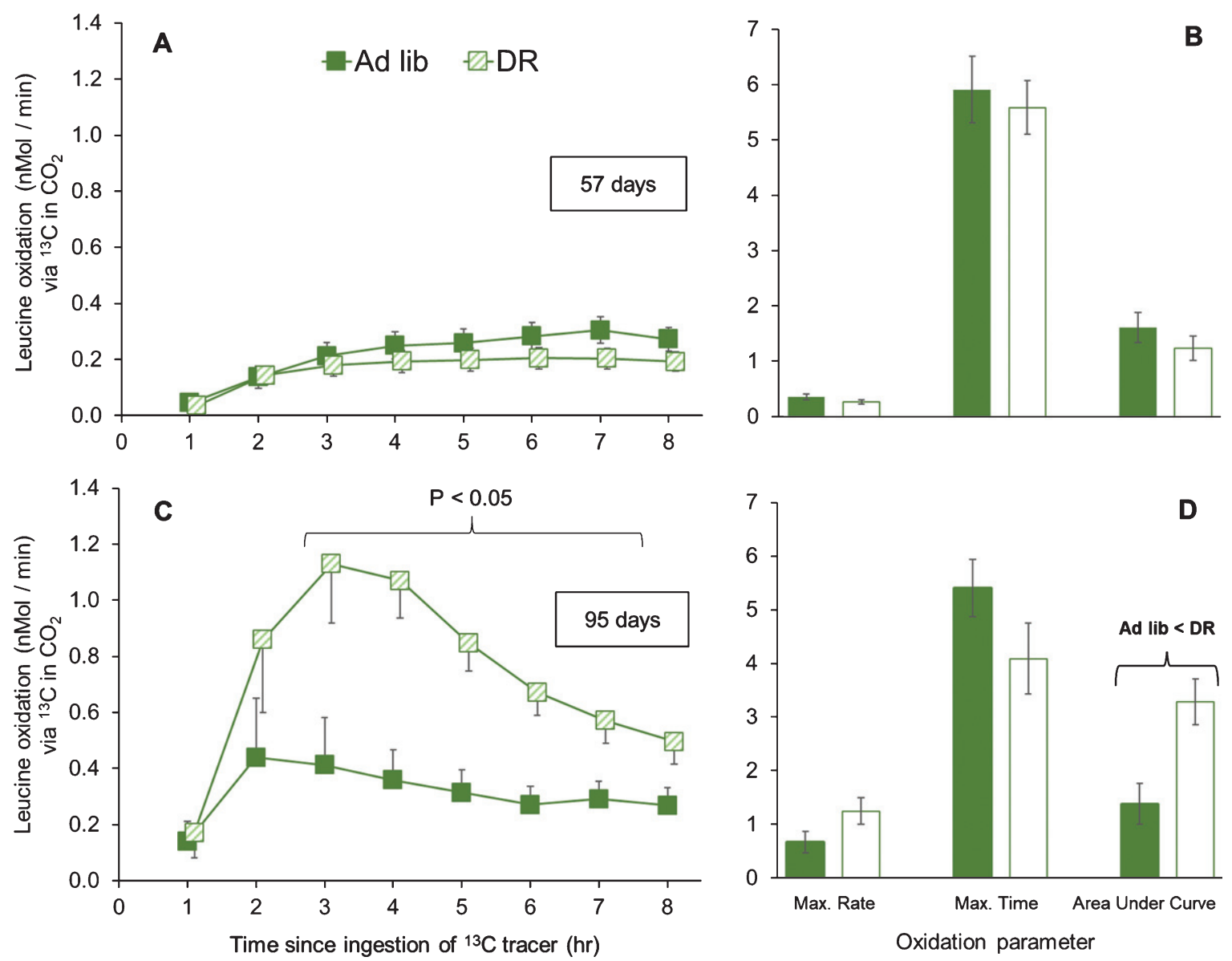

Fig. 7. Organismal oxidation of leucine upon ad libitum or DR lettuce feeding, in middle-aged female grasshoppers. Panels A and B show females at $\sim 57$ days of adulthood, during the reproductive period. After these measurements, each individual was switched to the other diet (i.e., ad libitum-fed grasshopper switched to DR, and DR-fed grasshoppers switched to ad libitum. Panels C and D show the same females, $\sim 40$ days after the diet switch, late in the reproductive period. Green bars show ad libitum-fed grasshoppers, while white bars show DR-fed grasshoppers. Life-extending dietary restriction of lettuce increased cumulative oxidation of leucine at $\sim 95$, but not at $\sim 57$ days of adulthood, as shown by two-way MANOVA. For panels B and D, maximum rates are nMol / min, maximum times are hours after tracer ingestion, and Area Under the Curve is in $\mathrm{nMol} / \mathrm{min} * 100$. Oxidation curve data were tested with one-way MANOVA with time as a dependent variable, and oxidation parameters were tested with one-way MANOVA. Data are offset to show one Standard Error bars.

leucine oxidation was significantly increased by the interaction of time after tracer ingestion and lettuce feeding (one-way MANOVA with time as a dependent variable; Pillai's Trace $\mathrm{F}_{7,20}=4.60 ; P=0.003$; Fig. 7C). Specifically, grasshoppers on DR lettuce had increased oxidation of leucine at 3 through $8 \mathrm{hr}$ (all $P<0.05$ ).

Similarly, for analysis of aspects of the oxidation curves, there were no significant effects for the trials at $\sim 57 \mathrm{~d}$ (Fig. 7B). However, for the trials at $\sim 95 \mathrm{~d}$, DR lettuce significantly increased leucine oxidation (one-way MANOVA; Pillai's Trace $\mathrm{F}_{3,24}=7.01$; $P=0.002$; Fig. 7D). Specifically, feeding on DR lettuce increased the AUC (i.e., cumulative leucine oxidation; $\left.\mathrm{F}_{1,26}=10.60 ; \quad P=0.003\right)$, marginally increased the maximum rate of leucine oxidation $(P=0.080)$, but did not affect the time at which the maximum rate of leucine oxidation occurred $(P=0.132)$.

\subsection{Dietary restriction can increase oxidation of leucine in young, but not middle-aged, adult males}

In Experiment 3, young adult males (median age 14 days into adulthood; total $n=12$ ) were tested on extreme dietary restriction (a 30\% diet). Leucine oxidation was not affected by the interaction of 

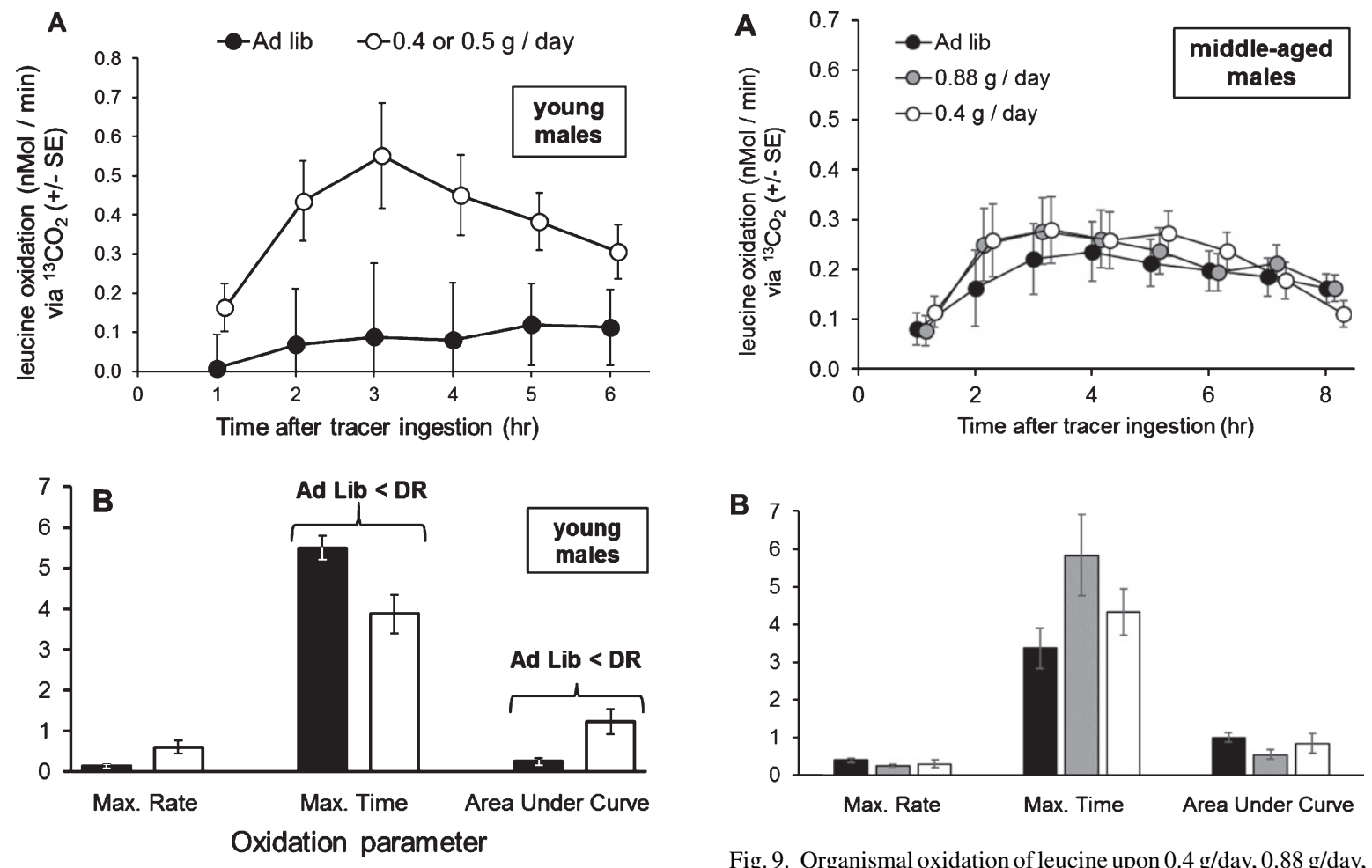

Fig. 8. In young adult male grasshoppers, organismal oxidation of leucine was increased upon a 30\% diet. A) The 30\% diet marginally increased oxidation of leucine from 2 to 5 hours after tracer ingestion. B) The $30 \%$ diet significantly decreased the time of occurrence of the maximum rate of oxidation (hours after tracer ingestion) and significantly increased the Area Under the Curve (nMol/min * 100), but did not change the maximum rate of oxidation $(\mathrm{nMol} / \mathrm{min})$. Oxidation curve data were tested with one-way MANOVA with time as a dependent variable, and oxidation parameters were tested with Student t-tests. Error bars show one Standard Error.

time after tracer ingestion and lettuce feeding (oneway MANOVA; Pillai's Trace $\mathrm{F}_{5,6}=1.73 ; P=0.262$; Fig. 8A). However, DR lettuce nearly significantly increased leucine oxidation at $2(P=0.064), 3$ $(P=0.073), 4(P=0.065)$, and $5(P=0.068)$ hours after tracer ingestion. These times encompass the period of greatest oxidation. For parameters of oxidation, DR lettuce increased the AUC (i.e., cumulative oxidation; two-tailed t-test; Bonferroni corrected $\alpha=0.017 ; \mathrm{t}_{11}=2.88 ; P=0.015$; Fig. $8 \mathrm{~B}$ ), decreased the time of occurrence of the maximum rate of leucine oxidation ( $\left.\mathrm{t}_{11}=2.85 ; P=0.016\right)$, but only marginally increased the maximum rate of leucine oxidation $\left(\mathrm{t}_{11}=2.65 ; P=0.023\right)$.

Middle-aged males (median age 97 days into adulthood; total $n=29$ ) were tested on a $0.4 \mathrm{~g}$ per day,

Fig. 9. Organismal oxidation of leucine upon $0.4 \mathrm{~g} /$ day, $0.88 \mathrm{~g} /$ day, or ad libitum lettuce feeding, in middle-aged male grasshoppers. A) Diet level did not affect leucine oxidation at any point tested. B) Diet level did not affect the maximum rate of leucine oxidation ( $\mathrm{nMol} / \mathrm{min}$ ), the time of occurrence of the maximum rate of leucine oxidation (hours after tracer ingestion), or the Area Under the Curve $(\mathrm{nMol} / \min * 100)$. Oxidation curve data were tested with one-way MANOVA with time as a dependent variable, and oxidation parameters were tested with one-way MANOVA. Error bars show one Standard Error.

$0.88 \mathrm{~g}$ per day, or ad libitum lettuce feeding. The feeding rate of ad libitum-fed males at middle age was not tested, so the degree of dietary restriction is not known. That said, the interaction of diet level and time did not affect leucine oxidation (one-way repeated-measures ANOVA; Pillai's Trace $\mathrm{F}_{14,42}=1.13 ; P=0.360$; Fig. 9A), and leucine oxidation was unchanged at all time points (all $P>0.25$ ). Similarly, diet level did not affect the maximum rate of oxidation, the time of occurrence of the maximum rate of oxidation, or the Area Under the Curve (one-way MANOVA; Pillai's Trace F6,32=1.18; $P=0.339$; Fig. 9B) in middle-aged males.

\section{Discussion}

Here we show that life-extending dietary restriction can increase the oxidation of BCAAs for 
production of ATP. The results that best support this conclusion are the highly significant increases in the cumulative rates of oxidation. While the oxidation of leucine was not always increased upon dietary restriction, the clearest overall view of the results is that dietary restriction that creates an energy deficit and increases hunger can increase oxidation of BCAAs. This should reduce the amount of BCAAs that could activate the mTOR pathway or reduce insulin sensitivity. In contrast to the results for dietary restriction, the predictions that supplementation of BCAAs would increase oxidation of BCAAs, feeding rate, or egg production, were not supported. This suggests that oxidation of BCAAs is not increased even when they are superfluous.

Previous work has shown that oxidation of BCAAs can increase in a catabolic state [35], such as fasting. However, life-extending dietary restriction is not starvation, but instead a rate of feeding that at least permits maintenance of weight and may allow weight gain at a reduced rate [e.g., 23, 24, 36, 37], yet likely creates an energy deficit [17]. Whether organismal oxidation of BCAAs increases upon dietary restriction (sensu, biology of aging) was previously unknown. Here we show that oxidation of each of the three BCAAs can increase upon dietary restriction, which should result in less BCAAs available to perturb health.

Male grasshoppers showed increased oxidation of leucine, but only at young ages and upon severely restricted diets. Old male grasshoppers are rarely hungry. Even when placed on a $60 \%$ diet (relative to other similar-aged males), they do not eat voraciously. Females on a chronic $60 \%$ diet eat immediately upon being offered their daily food, and typically eat $\sim 20 \%$ of their body mass in the first feeding bout. In contrast, old male grasshoppers on a chronic $60 \%$ diet sometimes do not eat any of their meal immediately upon being offered food. In Experiment 3 of the present paper, young adult male grasshoppers on a $30 \%$ diet ate immediately and completed the entire meal that was offered. These males on a $30 \%$ diet showed increased leucine oxidation, consistent with the notion that males, as well as females, when sufficiently hungry can increase oxidation of BCAAs.

We see increased oxidation of amino acids upon dietary restriction as common but not universal, and not necessarily unique to the BCAAs. Data on alanine and cysteine support this view. Oxidation of alanine, a non-essential amino acid, nearly significantly increased upon dietary restriction (similar to leucine in Experiment 1; see Supplemental Figure 2). However, cysteine oxidation was not affected by dietary restriction, and was very low throughout the trial. Notably, the increased organismal oxidation upon dietary restriction does not need to be special to the BCAAs to have a role in life-extension. BCAAs have special physiological roles, such as mTOR activation, insulin insensitivity, and hyperphagia (see discussion of [38] below), so special rates of oxidation are not needed to support our broad hypothesis.

Leucine is preferentially metabolized by skeletal muscle, where it can be completely oxidized, in contrast to most other amino acids [39]. This is especially true during fasting [35]. While we did not isolate muscle in our experiments, because of the propensity of leucine to oxidize in muscle, and the increase of oxidation in the muscle upon fasting, it is likely that ATP production in muscle contributes prominently to the increases in organismal oxidation observed here. Whether life-extending dietary restriction increases oxidation of BCAAs in muscle relative to other tissues, as diabetes in mice does [20], is undetermined.

Is the degree of increased oxidation of BCAAs sufficient to contribute to reduced activation of the mTOR pathway, or increase insulin sensitivity? In these trials, a small portion of the ingested ${ }^{13} \mathrm{C}$ tracer is oxidized by the end of the trial, sometimes $\sim 5 \%$. This represents the BCAAs that are oxidized immediately, probably before incorporation into a protein; additional BCAAs would be oxidized later. Further, similar magnitudes of ${ }^{13} \mathrm{C}$-amino acid recovery in the breath have been reported in other insects [40, 41], amphibians [42], reptiles [43], birds [44], and mammals [45]. Does this reduction in available BCAAs due to mild (but often statistically significant) increases in oxidation contribute to health? Our view is that changes in oxidation rates may contribute to healthspan upon chronic dietary restriction, but not upon acute dietary restriction [which can reduce mortality rate; see 46]. To address this question, expression of enzymes involved in BCAAs oxidation could be manipulated. Branched-chain alpha-keto dehydrogenase is involved in oxidation of all three BCAAs [e.g., 39]. This enzyme would need to be overexpressed to a degree that results in similar levels of oxidation of BCAAs as seen upon dietary restriction. If this overexpression decreased mTOR activation (as indicated by increased S6-kinase phosphorylation), then increased oxidation of BCAAs upon dietary restriction would seem likely to contribute to healthspan. 
What causes the great variability in leucine oxidation, in comparison to isoleucine or valine oxidation? In Experiment 1, leucine oxidation increased 50\% upon dietary restriction, but this was not statistically significant, in part due to high inter-individual variability in the levels of leucine oxidation. One hypothesis for this variation is that the degree of leucine oxidation varies with the clutch cycle. In lubber grasshoppers, the levels of hormones and hemolymph proteins supporting egg production all peak about $14 \mathrm{~d}$ before laying, regardless of diet [47]. Middle-aged lubber grasshoppers fed ad libitum produce multiple large clutches, one about every $20 \mathrm{~d}$ [24]. Given the cycling in the factors underlying egg production, we hypothesized that oxidation of leucine might also vary throughout the clutch cycle. However, this hypothesis was not supported by the data. There was no association of the maximum level of leucine oxidation and the time since the most recent oviposition.

The leucine oxidation results for Experiment 2 at $57 \mathrm{~d}$ deviate from the other data in the study. These same individuals (after switching from ad libitum to DR lettuce, or visa versa) later showed more typical results, with higher leucine oxidation upon dietary restriction. This $57 \mathrm{~d}$ trial not only failed to show increased oxidation upon dietary restriction, but also showed much lower levels of oxidation than other trials. We have not identified why this trial deviated from the others, so we present the data here, but we have eliminated some possible errors. First, the samples had sufficient $\mathrm{CO}_{2}$ for measurement of ${ }^{13} \mathrm{C}$; this trial had an average $\left[\mathrm{CO}_{2}\right]$ of $1.4 \%$, and the Experiment 3 trial with young males had an average $\left[\mathrm{CO}_{2}\right]$ of $0.9 \%$ and ${ }^{13} \mathrm{C}$ was also measurable. Second, the samples showed a steady increase in ${ }^{13} \mathrm{CO}_{2}$. Between each $1 \mathrm{hr}$ collection period, the collection syringe was purged. We tested blank samples immediately after purging the syringe, and ${ }^{13} \mathrm{CO}_{2}$ was undetectable. This procedure should allow no contamination between samples. Third, in previous studies we have shown that females on dietary restriction have the same amount of undigested food in the gut as do females on ad libitum lettuce. This minimizes concern that the low maximum level of oxidation is due to reduced absorption.

We used chronic force-feeding to test whether superfluous dietary BCAAs results in increased oxidation of BCAAs. Confidence that our BCAAs supplementation was effective is supported by the hemolymph levels of isoleucine, which were 2-fold higher in DR \& BCAAs grasshoppers than in DR \& buffer grasshoppers. Further, this same trend exists for both leucine and valine, although not statistically significant $(\mathrm{P} \sim 0.10)$. Taken together, these data support the notion that the chronic force-feedings altered internal levels of BCAAs.

The dietary supplementation of all three BCAAs did not alter organismal oxidation of each individual BCAA. The outcome that oxidation of BCAAs did not increase even when hemolymph levels were higher (cf., dietary restriction lettuce \& BCAA vs. dietary restriction lettuce \& buffer) suggests that superfluous levels of BCAAs itself do not increase their oxidation. This further supports the conclusion that increases in oxidation of BCAAs upon dietary restriction were due to an energy deficit, not due to increased availability of the BCAAs. This is also consistent with the view that increased oxidation upon life-extending dietary restriction is not special to the BCAAs, but likely occurs for several amino acids.

Supplementation of BCAAs did not alter feeding rate. Solon-Biet and colleagues [38] showed that, in mice, increased dietary BCAAs in turn increased feeding about $20 \%$. The hyperphagia in mice was due to reduced tryptophan and serotonin levels. In our study, hemolymph tryptophan levels were not altered by BCAAs supplementation (data not shown; MANOVA; $\left.\mathrm{F}_{1,23}=0.09 ; P=0.764\right)$, so it is unlikely that altered amino acid levels led to reduced serotonin levels in grasshoppers. On the other hand, Juricic and colleagues [12] showed that, in flies, greatly reduced dietary BCAAs (the opposite of the dietary treatment used in mice) increased feeding about 2-fold. Near as we can tell, extreme levels of BCAAs in the diet (whether low or high) might increase feeding. It may be that the supplementation of BCAAs in our study was not extreme enough to cause hyperphagia, especially because our diets included lettuce.

Egg production was not increased upon supplementation of BCAAs. This result is consistent with predictions of Piper et al. (2017), which suggests that a diet matched to the proteins coded for by the exome reduce feeding rate yet increase development rate. The exome for lubber grasshoppers is not available, but Piper and colleagues [11] show that the amino acid profile of yolk protein is a good representative for the amino acid profile of the exome. In comparing the amino acid content of Romaine lettuce to the amino acid content of grasshopper vitellin, the essential amino acid lysine is more limiting than is isoleucine. This suggests that without simultaneous supplementation of lysine, supplementation of the BCAAs will not increase grasshopper egg production. 
Overall, we observed effects of dietary restriction, but not supplementation of BCAAs, on oxidation. Consistent with this, other physiological parameters were also affected by dietary restriction, but not by supplementation of BCAAs, in Experiment 1. First, levels of hexameric storage proteins in the hemolymph were decreased upon dietary restriction, but were not increased upon supplementation of BCAAs, despite the fact that these hexamerins are composed of nearly 20\% BCAAs (Supplemental Table 1). Second, percentages of nitrogen and carbon in the fat body were affected by dietary restriction, but not by supplementation of BCAAs (Supplemental Figure 3). In particular, fat body nitrogen was increased upon dietary restriction, perhaps due to less lipid storage. Fat body carbon was decreased upon dietary restriction, perhaps due to reduced glycogen storage.

Taken together, our results support the hypothesis that dietary restriction creates a sufficient energy deficit to increase oxidation of the BCAAs, even during the protein-demanding period of egg production. This should reduce the levels of available BCAAs, which could contribute to improved healthspan in several ways. Oxidation of BCAAs may be an underappreciated contributor to life-extension via dietary restriction.

\section{Acknowledgments}

We thank Ryan Koch and Tim Kelting for assisting with alanine and cysteine oxidation experiments, and James Kellenberger for reviewing the manuscript.

\section{Funding}

Supported by NIA award 1R15AG050218-01A1 to JDH. The authors claim no conflicts of interest.

\section{Conflict of interest}

The authors have no conflict of interest to report.

\section{Supplementary material}

The supplementary material is available in the electronic version of this article: https://dx.doi.org/ 10.3233/NHA-190073

\section{References}

[1] Lee KP, Simpson SJ, Clissold FJ, Brooks R, Ballard JWO, Taylor PW, Soran N, Raubenheimer D. Lifespan and reproduction in Drosophila: New insights from nutritional geometry. Proc Nat Acad Sci. 2008;105(7):2498-2503.

[2] Solon-Biet SM, McMahon AC, Ballard JWO, Ruohonen K, Wu LE, Cogger VC, Warren A, Huang X, Pichaud N, Melvin RG, Gokarn R, Khalil M, Turner N, Cooney GJ, Sinclair DA, Raubenheimer D, Le Couteur DG, Simpson SJ. The ratio of macronutrients, not caloric intake, dictates cardiometabolic health, aging, and longevity in ad libitum-fed mice. Cell Met. 2014;19:418-430.

[3] Solon-Biet SM, Mitchell SJ, de Cabo R, Raubenheimer D, Le Couteur DG, Simpson SJ. Macronutrients and caloric intake in health and longevity. J Endocrinol. 2015;226: R17-R28.

[4] Speakman JR, Mitchell SE, Mazidi M. Calories or protein? The effect of dietary restriction on lifespan in rodents in explained by calories alone. Exp Geron. 2016;56:28-38.

[5] Grandison RC, Piper MDW, Partridge L. Amino-acid imbalance explains extension of lifespan by dietary restriction in Drosophila. Nature 2009;462(7276):1061-1064.

[6] Brown-Borg HM, Buffenstein R. Cutting back on the essentials: Can manipulating intake of specificamino acids modulate health and lifespan? g intake of specific amino acids modulate health and lifespan? Age Res Rev. 2017;39: 87-95.

[7] Orentreich N, Matias JR, DeFelice A, Zimmerman JA. Low methionine ingestion by rats extends life span. J Nutrition. 1993;123(2):269-274.

[8] Lee BC, Kaya A, Ma S, Kim G, Gerashchenko MV, Yim SH, Hu Z, Harshman LG, Gladyshev VN. Methionine restriction extends lifespan of Drosophila melanogaster under conditions of low amino acid status. Nat. Comm. 2014;5: 3592.

[9] Kabil H, Kabila O, Banerjeec R, Harshman LG, Pletcher SD. Increased transsulfuration mediates longevity and dietary restriction in Drosophila. Proc. Nat. Acad. Sci. 2011;108(40):16831-16836.

[10] Hine C, Harputlugil E, Zhang Y, Ruckenstuhl C, Lee BC, Brace L, Longchamp A, Treviño-Villarreal JH, Mejia P, Ozaki CK, Wang R, Gladyshev VN, Madeo F, Mair WB, Mitchell JR. Cell 2015;160(1-2):132-44.

[11] Piper MDW, Soultoukis GA, Blanc E, Mesaros A, Herbert SL, Juricic P, He X, Atanassov I, Salmonowicz H, Yang M, Simpson SJ, Ribeiro C, Partridge L. Matching dietary amino acid balance to the in silico-translated exome optimizes growth and reproduction without cost to lifespan. Cell Met. 25:610-621.

[12] Juricic P, Grönke S, Partridge L. Branched-chain amino acids have equivalent effects to other essential amino acids on lifespan and ageing-related traits in Drosophila. J Geron Biol. Sci. 2019; doi: 10.1093/gerona/glz080.

[13] Mansfeld J, Urban N, Priebe S, Groth M, Frahm C, Hartmann N, Gebauer J, Ravichandran M, Dommaschk A, Schmeisser S, Kuhlow D, Monajembashi S, Bremer-Streck S, Hemmerich P, Kiehntopf M, Zamboni N, Englert C, Guthke R, Kaleta C, Platzer M, Sühnel J, Witte OW, Zarse K, Ristow M. Branched-chain amino acid catabolism is a conserved regulator of physiological ageing. Nat Comm. 2015;6: 10043. 
[14] Fontana L, Cummings NE, Arriola Apelo SI, Neuman JC, Kasza I, Schmidt BA, Cava E, Spelta F, Tosti V, Syed FA, Baar EA, Veronese N, Cottrell SE, Fenske RJ, Bertozzi B, Brar HK, Pietka T, Bullock AD, Figenshau RS, Andriole GL, Merrins MJ, Alexander CM, Kimple ME, Lamming DW. Decreased consumption of branched chain amino acids improves metabolic health. Cell Rep. 2016;16(2):520-530.

[15] Wu Q, Zhang Y, Xu J, Shen P. Regulation of hunger-driven behaviors by neural ribosomal S6 kinase in Drosophila. Proc Nat Acad Sci. 2005;102(37):13289-13294.

[16] Minor RK, Chang JW, de Cabo R. Hungry for life: How the arcuate nucleus and neuropeptide $\mathrm{Y}$ may plan a critical role in mediating the benefits of calorie restriction. Mol Cell Endocrinol. 2009;299(p1):79-88.

[17] Stenesen D, Suh JM, Seo J, Yu K, Lee K.-S., Kim J.-S., Min K.-J., Graff JM. Adenosine nucleotide biosynthesis and AMPK regulate adult life span and mediate the longevity benefit of caloric restriction in flies. Cell Met. 2013;17:101112.

[18] Saxton RA, Sabatini DM. mTOR signaling in growth, metabolism, and disease. Cell 2017;168:960-976.

[19] Kennedy BK, Lamming DW. The mechanistic target of rapamycin: The grand conducTOR of metabolism and aging. Cell Met. 2016;23:990-1003.

[20] Neinast MD, Jang C, Hui S, Murashige DS, Chu Q, Morscher RJ, Li X, Zhan L, White E, Anthony TG, Rabinowitz JD, Arany Z. Quantitative analysis of the whole-body metabolic fate of branched-chain amino acids. Cell Met. 2019;29; $1-13$.

[21] Cummings NE, Williams EM, Kasza I, Konon EN, Schaid MD, Schmidt BA, Poudel C, Sherman DS, Yu D, Arriola Apelo SI, Cottrell SE, Geiger G, Barnes ME, Wisinski JA, Fenske RJ, Matkowskyj KA, Kimple ME, Alexander CM, Merrins MJ, Lamming DW. Restoration of metabolic health by decreased consumption of branched-chain amino acids. J Physiol. 2017;596(14):623-645.

[22] Lee H.-Y., Lee S.-H., Min K.-J. Insects as a model system for aging studies. Entomol Res. 2015;45:1-8.

[23] Hatle JD, Wells S, Fuller LE, Allen IC, Gordy LJ, Melnyk $\mathrm{S}$ and Quattrochi J. Calorie restriction and late-onset calorie restriction extend lifespan but do not alter protein storage in female grasshoppers. Mech Age Devel. 2006;127:883-891.

[24] Drewry MD, Williams JM, Hatle JD. Life-extending dietary restriction and ovariectomy result in similar feeding rates but different physiological responses in grasshoppers. Exp Geron. 2011;46:781-786.

[25] Chapman RF. The Insects: Structure and Function. 4th ed. Cambridge: Cambridge University Press. 1998.

[26] Hatle JD, Awan A, Nicholas J, Koch R, Vokrri JR, McCue MD, Williams CM, Davidowitz G, Hahn DA. Life-extending dietary restriction and ovariectomy each increase leucine oxidation and alter leucine allocation in grasshoppers. Exp Geron. 2017;96:155-161.

[27] Whitman DW, Blum MS, Alsop DW. Allomones: Chemicals for defense. In: Evans DL, Schmidt JO, editors. Insect defenses: Adaptive mechanisms and strategies of prey and predators. Albany: State University of New York Press; 1990. p. 289.

[28] Gruber H, Wessels W, Boynton P, Xu J, Wohlgemuth S, Leeuwenburgh C, Qi W, Austad SN, Schaible R, Philipp EE. Age-related cellular changes in the long-lived bivalve A. islandica. Age (Dordr). 2015;37(5):90.
[29] Hatle JD, Kellenberger JW, Viray E, Smith AM, Hahn DA. Life-extending ovariectomy in grasshoppers increases somatic storage, but dietary restriction with an equivalent feeding rate does not. Exp Geron. 2013;48:966-972.

[30] Self Nutrition Data. Condé Nast. 2018. [updated 2018 May 25; cited 2019 July 16]. Available from https://nutritiondata. self.com/facts/vegetables-and-vegetable-products/2475/2.

[31] McCue MD, Guzman RM, Passement CA. Digesting pythons quickly oxidize the proteins in their meals and sae the lipids for later. J Exp Biol. 2015;218:2089-2096

[32] McCue MD, Boardman L, Clusella-Trullas S, Kleynhans E, Terblanche JS. The speed and metabolic cost of digesting a blood meal depends on temperature in a major disease vector. J Exp Biol. 2016;219:1893-1902.

[33] Burmester T. Origin and evolution of arthropod hemocyanins and related proteins. J Comp Physio.1 B 2002;172(2):95-107.

[34] Hathaway M, Hatle JD, Li S, Ding X, Barry T, Hong F, Wood $\mathrm{H}$, Borst DW. Characterization of hexamerin proteins and their mRNAs in the adult lubber grasshopper: the effects of nutrition and juvenile hormone on their levels. Comp Biochem Physiol. A 2009;154:323-332.

[35] Brestenský M, Nitrayová S, Patráš P, Heger J, Nitray J. Branched-chain amino acids and their importance in nutrition. J Microbiol Biotech Food Sci. 2015;5(2):197-202.

[36] Anson RM, Guo Z, de Cabo R, Iyun T, Rios M, Hagepanos A, Ingram DK, Lane MK, Mattson MP. Intermittent fasting dissociates beneficial effects of dietary restriction on glucose metabolism and neuronal resistance to injury from caloric intake. Proc Nat Acad Sci. 2003;100(10): 6216-622.

[37] Sohal RS, Forster MJ. Caloric restriction and the aging process: A critique. Free Radic. Biol. Med. 2014;73:366-382.

[38] Solon-Biet SM, Cogger VC, Pulpitel T, Wahl D, Clark X, Bagley EE, Gregorious GC, Senior AM, Wu Q-P, Brandon AE, Perks R, O'Sullivan J, Koay YC, Bell-Anderson K, Kebede M, Yau B, Atkinson C, Svineng G, Dodgson T, Wali JA, Piper MDW, Juricic P, Partridge L, Rose AJ, Raubenheimer D, Cooney GJ, Le Couteur DG, Simpson SJ. Branched-chain amino acids impact health and lifespan indirectly via amino acid balance and appetite control. Nat Met. https://doi.org/10.1038/s42255-019-0059-2.

[39] Gropper SS, Smith JL, Carr TP. Advanced nutrition and human metabolism. 7th ed. Boston: Cengage Learning; 2013.

[40] Williams CM, McCue MD, Sunny NE, Szejner-Sigal A, Morgan TJ, Allison DB, Hahn DA. Cold-adaptation increases rates of nutrient flow and metabolic plasticity during cold exposure in Drosophila melanogaster. Proc Biol Sci. 2016;283(1838):20161317.

[41] Levin E, McCue MD, Davidowitz G. Sex differences in the utilization of essential and non-essential amino acids in a Lepidoptera. J Exp Biol. 2017;220(15):2743-2747.

[42] Kirschman LJ, McCue MD, Boyles JG, Warne RW. Exogenous stress hormones alter energetic and nutrient costs of development and metamorphosis. J Exp Biol. 2017;220(18):3391-3397.

[43] Plasman M, McCue MD, Reynoso VH, Terblanche JS, Clusella-Trullas S. Environmental temperature alters the overall digestive energetics and differentially affects dietary protein and lipid use in a lizard. J Exp Biol. 2019;222(6); doi: 10.1242/jeb. 194480

[44] McCue MD, McWilliams SR, Pinshow B. Ontogeny and nutritional status influence oxidative kinetics of exogenous 
nutrients and whole-animal bioenergetics in zebra finches, Taeniopygia guttata. Physiol Biochem Zool. 2011;84(1):3242.

[45] McCue MD, Voigt CC, Jefimow M, Wojciechowski MS. Thermal acclimation and nutritional history affect the oxidation of different classes of exogenous nutrients in Siberian hamsters, Phodopus sungorus. J Exp Biol. 2014;321(9): 503-514.
[46] Mair W, Goymer P, Pletcher SD, Partridge L. Demography of dietary restriction and death in Drosophila. Science 2003;301:1731-1733.

[47] Hatle JD, Borst DW, Juliano SA. Plasticity and canalization in the control of reproduction in the lubber grasshopper. Integ Comp Biol. 2004;43:635-645. 